\title{
IoT based Heart Attack Detection, Heart Rate and Temperature Monitor
}

\author{
Gowrishankar S., PhD \\ Professor \\ Department of CSE \\ B.M.S. College of Engineering \\ Bangalore, Karnataka, India
}

\author{
Prachita M. Y. \\ Student \\ Department of CSE \\ B.M.S. College of Engineering \\ Bangalore, Karnataka, India
}

\author{
Arvind Prakash \\ Project Engineer \\ Livikodez \\ Bangalore, Karnataka, India
}

\begin{abstract}
The Internet of Things (IoT) is inter communication of embedded devices using networking technologies. The IoT will be one of the important trends in future, can affect the networking, business and communication. In this paper, proposing a remote sensing parameter of the human body which consists of pulse and temperature. The parameters that are used for sensing and monitoring will send the data through wireless sensors. Adding a web based observing helps to keep track of the regular health status of a patient. The sensing data will be continuously collected in a database and will be used to inform patient to any unseen problems to undergo possible diagnosis. Experimental results prove the proposed system is user friendly, reliable, economical.
\end{abstract}

\section{Keywords}

IoT, Heart rate sensors, Health monitoring, Health diagnosis.

\section{INTRODUCTION}

The Internet of Things (IoT) is inter communication of embedded devices using networking technologies. The IoT will be one of the important trends in future, can affect the networking, business and communication. IoT typically expected to propose the advanced high bandwidth connectivity of embedded devices, systems and services which goes beyond machine-to-machine (M2M) context. The advanced connectivity of devices aide in automation is possible in nearly all field.

Everyone today is so busy in their lives, even they forget to take care of their health. By keeping all these things in minds, technology really proves to be an asset for an individual. With the advancement in technology, lots of smart or medical sensors came into existence that continuously analyzes individual patient activity and automatically predicts a heart attack before the patient feels sick. Therefore, identifying the correct sensors is important.

In the medical field, nowadays patient take actively part in collecting and reviewing their reports. In this digitized world, various wireless communication standards have allowed the sensor to develop from traditional forms i.e. require active patient participation to passive form i.e. require no need for patient participation.

Today's large number of passive sensors are used that constantly monitor individual patient essential signs and store that data or share it wirelessly with Human-Healthcare professionals [1] [2] [3]. By combining analytics and sensor data, reports are made that describe the early health condition of the patient [4]. Depending on the requirement various types of sensors are being deployed.

Recently, the research of Human-Health monitoring systems has moved from basic reasoning of wearable sensor [5] readings to the advanced level of data processing to give more information that is valuable to the end users either to doctor or to patient.

Habitual diseases have a powerful influence on HumanHealthcare where cost of curing chance of attack is natural among people. Changes in analytical structure and dearth of health and social care forces to study new modernization technique, which could be a help to these obstacles.

Elderly people need to make regular visit to the doctor for their health signs test results. Observing on regular basis of essential signs is compulsory as they are main signs of well-being of one's individual health [1].

These vital signs include,

a. Pulse rate

b. Body temperature

The goal is to develop a low power, more reliable, nonintrusive, are the essential signs monitor which gather information on the body and send the parameters through wireless technology [6].

There must be a proper method of transmission and to display the signal after the data is processed. RPM is a one of the main technology which will help us to monitor the patients who are not in reach of common clinical settings, which will increase the access to care and reduce the cost of delivering Human-Healthcare.

In many developing countries, Human-Healthcare is frequently defined as a major problem. i.e., almost as much as $8 \%$ of the population who can has access to $20 \%$ of the country's medical resources. This inability to access proper HumanHealthcare for rural populations paired with rapidly increasing cardiovascular disease rates poses a serious problem.

In this paper, proposing a remote monitoring and sensing parameter of the human body which consists of pulse and temperature. The parameters that are used for sensing and observing will send the data through wireless sensors. Adding a web based observing helps to keep track of the regular health status of a patient. The sensing data will be collected in database continuously and will be used to inform patient to any unseen problem to undergo possible diagnosis.

\section{PROPOSED SYSTEM}

The introduced Human-Wellbeing checking/watching system includes patients, Human-Wellbeing observing units, cloud for information maintenance and secure [7]. With the assistance of some equipment units, different sensors and gadgets with web association [8]. The figure 1 shows the complete architecture of proposed system. The system functionality is divided into major three modules; they are:

1) Sensing module

2) The Main module and

3) Interaction module. 
Sensing module senses the state of the patient using sensors. Main module is for gathering information via sensing module and for data storing into the cloud. And the user (doctors or patient or monitor) interacting with the system via interaction module.

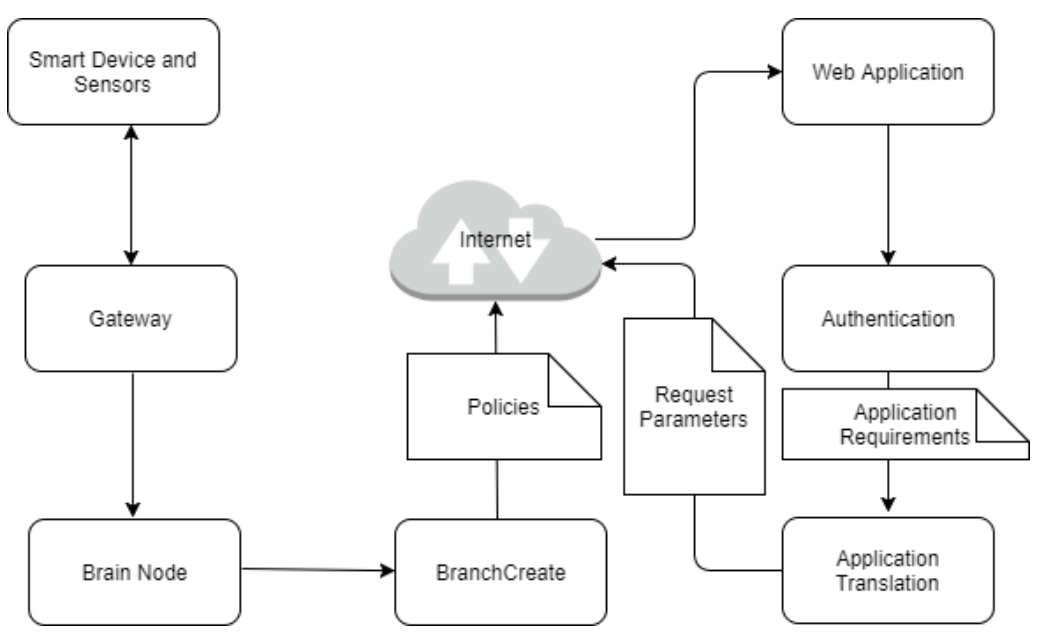

Fig. 1. System Architecture of the Proposed System

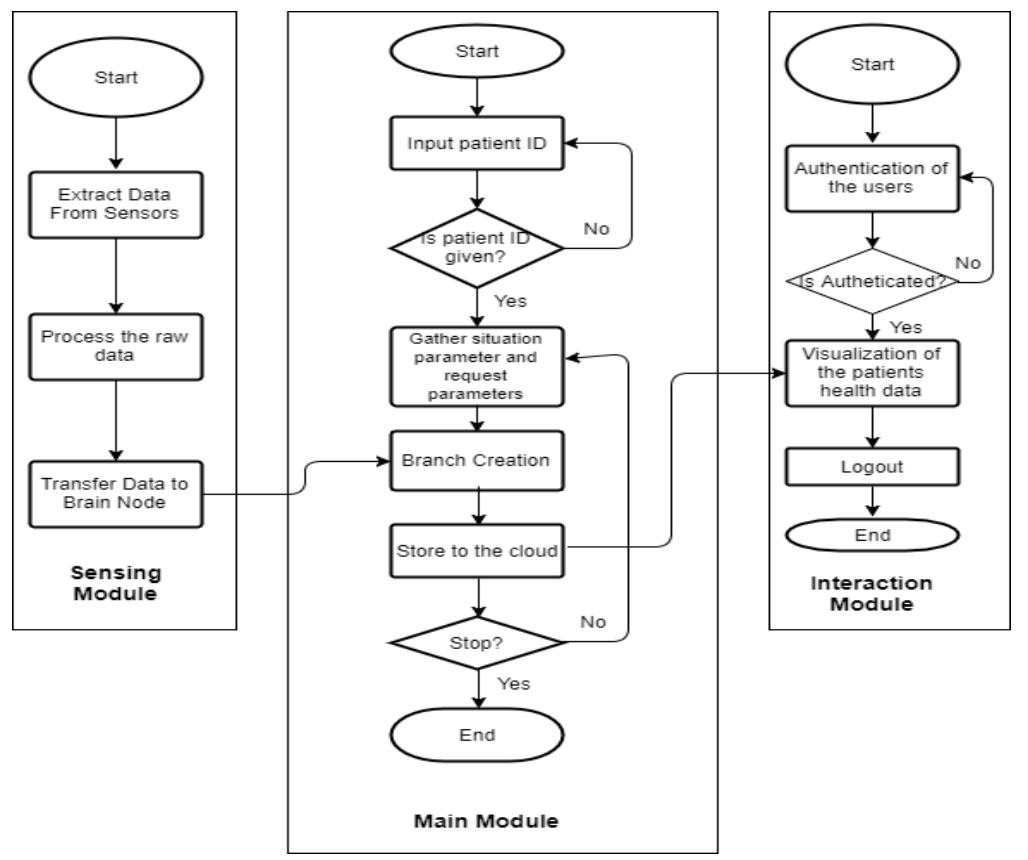

Fig. 2. System Flowchart of the Proposed System

\subsection{Sensing Module}

Sensing module consists of some sub-modules called sensors node and brain node. Pulse sensor and temperature sensor are attached to the patient's body for perceiving health data from the patient. In other words, these sensors collect the readings from the patient. The Sensors which are attached to the patient's body are then interfaced to the Arduino [9]. And readings are transmitted to brain node through gate-way. This Gateway acts as the intermediate between the sensors and the brain node. The sensors which collect the readings store the values in their flash memory. This stored data is transmitted to the brain node at regular intervals, the data is sent only at regular intervals because during this time intervals the values obtained which can either analog or digital will be converted into best suitable for human understanding. All collected data are stored into brain node. The data stored in the brain node is then sent to the main module by using the Wi-Fi module interfaced to the Arduino After some time the brain node transmits a set of data to main module. In the sensing module, all the sensors are interfaced to the Arduino. The sensors being

- Pulse Sensor

- Temperature Sensor

- Wi-Fi Module

\section{Pulse Sensor}

Pulse Sensor Amped is a plug-and-play heart-rate sensor for Arduino compatibles. Pulse Sensor Amped works with either a $3 \mathrm{~V}$ or $5 \mathrm{~V}$. There are 4 steps involved in interfacing the pulse sensor to the Arduino and to the computer.

\section{Temperature Sensor}

LM35 is an analog, linear temperature sensor whose output voltage varies linearly with change in temperature. The figure 3 shows the pin out connection of LM35. 


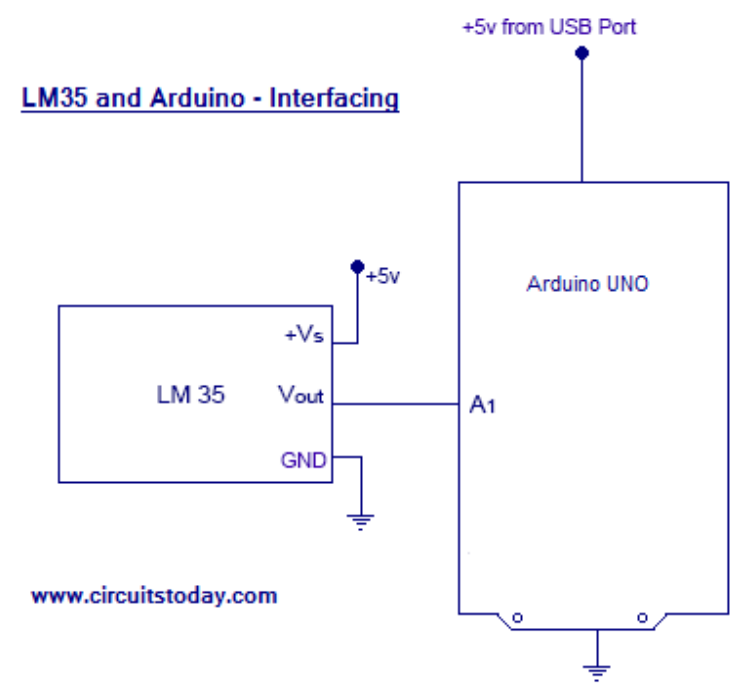

Fig. 3. Pin connection of Temperature Sensor to Arduino

\section{Wi-Fi Module}

Connect ESP8266 module with Arduino. ESP8266 runs on $3.3 \mathrm{~V}$, so need to power from the $3.3 \mathrm{~V}$ output of Arduino. Connect VCC and CH_PD of ESP8266 module to 3.3V output of Arduino and the ground of ESP8266 to the ground of Arduino. The RX pin of the ESP8266 is not $5 \mathrm{~V}$ tolerant so, need to reduce the $5 \mathrm{~V}$ TX output of Arduino to $3.3 \mathrm{~V}$ using voltage dividing resistors. Here, using three $1 \mathrm{~K} \Omega$ resistors connected in series for that. So, connect pin 10 (TX) of Arduino to RX of ESP8266 module via voltage dividing resistors. Can directly connect TX pin of ESP8266 to 9th (RX) of Arduino as it will detect $3.3 \mathrm{~V}$ as logic HIGH according to TTL Voltage specification [10].

\subsection{The Main Module}

The sub modules which live through internet in the main module are branch creation and storage or data base. The data stored in permanent storage unit called data base. These data are distributed in many different branches in branch node. Therefore, the creation of a database is the first step in the main module and these branch nodes are collected in database through the internet.

\subsection{Interaction Module}

In interaction module consists of su-module they are: Application translation, situation acquisition. The application requirements are patient's information, the situation parameter is:

$$
\begin{array}{ll}
\text { - } & \text { Date } \\
\text { - } & \text { Time } \\
\text { - } & \text { Location etc. }
\end{array}
$$

Interaction system will give permission to communicate with system for only authenticated user, i.e., user information should match with the data stored in database.

ThingSpeak is an open source IoT application with HTTP API which can store and retrieve data from the 'things' via Internet or Intranet. By using this anyone can easily create a network of things which can be used for applications like logging, tracking, analytics etc. First, open the ThingSpeak Website and create an account

The figure 2 shows the flow diagram of proposed system, there are three modules in proposed system as discussed above in the chapter. Initially, the main module collects the data through the brain node to monitoring system. The proposed system will create branch and it will store data to cloud through internet if patient ID is already provided, if patient Id is not there in database, then it will wait for input from patient for their ID and intern it stores the data in cloud. Mean-while, system will stop storing data to the database when stop command is given. The patient data can be seen from authenticated users only in interaction module through internet, the authenticated user must re-verify if session expires.

\section{EXPERIMENTAL RESULT}

The results are to illustrate that all the modules are operating correctly without any data loss and each sub-module in all modules are performing their function. The Pulse Sensor and Temperature Sensor of sensing module should extract the accurate readings and should be able to send the data to the Arduino. The Wi-Fi module which is also a part of the sensing module must send the values to the server without any delay and without any data loss. The Server must store all the data sent by the Wi-Fi module and display the same on the Web Server.

\subsection{Experimental Results on Pulse Sensor}

After checking all the pin connections and adding the library to the Arduino IDE and uploading the source code, run the code. LCD display will be turned on to display the Human-HumanHeart rate reading obtained by the Pulse Sensor. Place your index finger on the front side of the pulse sensor, you should see LED1 (red) glow in time with your Human-Heartbeat when you place your finger on the sensor. Place your finger on the sensor lightly till you get a read on the LCD or serial monitor that shows signal is already transmitted. You will be able to get the reading on both serial monitor and LCD.

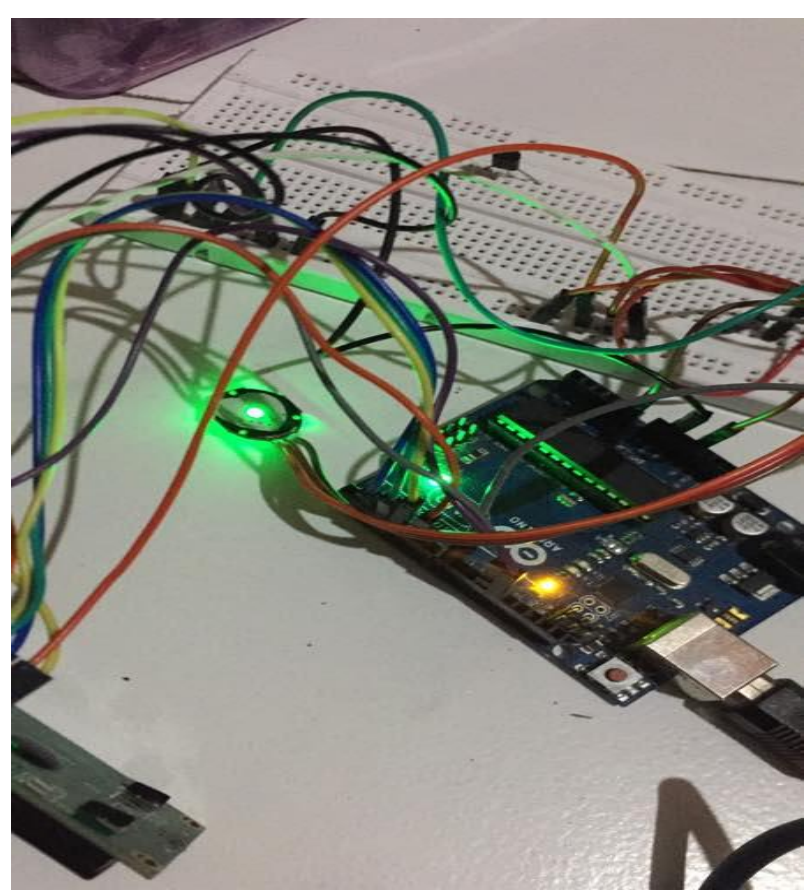

Fig. 4. Working of the Pulse Sensor

The different test cases have been considered for the HumanHuman-Heart rate of a patient are

- When the patient is sitting idle

- When the patient is a sleep

- When the patient is running

- Also, when a patient has undergone an abnormal Human-Heartbeat 


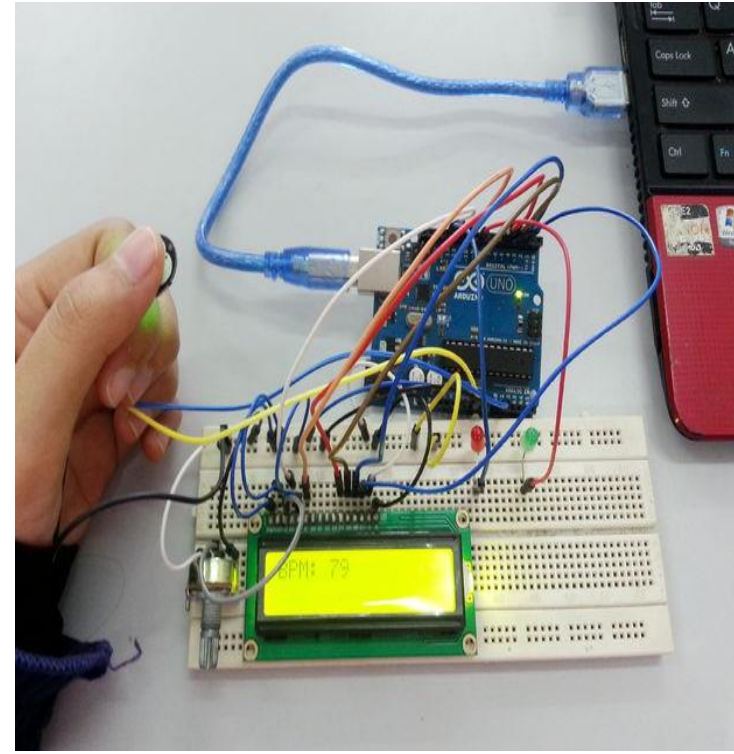

Fig. 5. pulse rate Output display on LCD

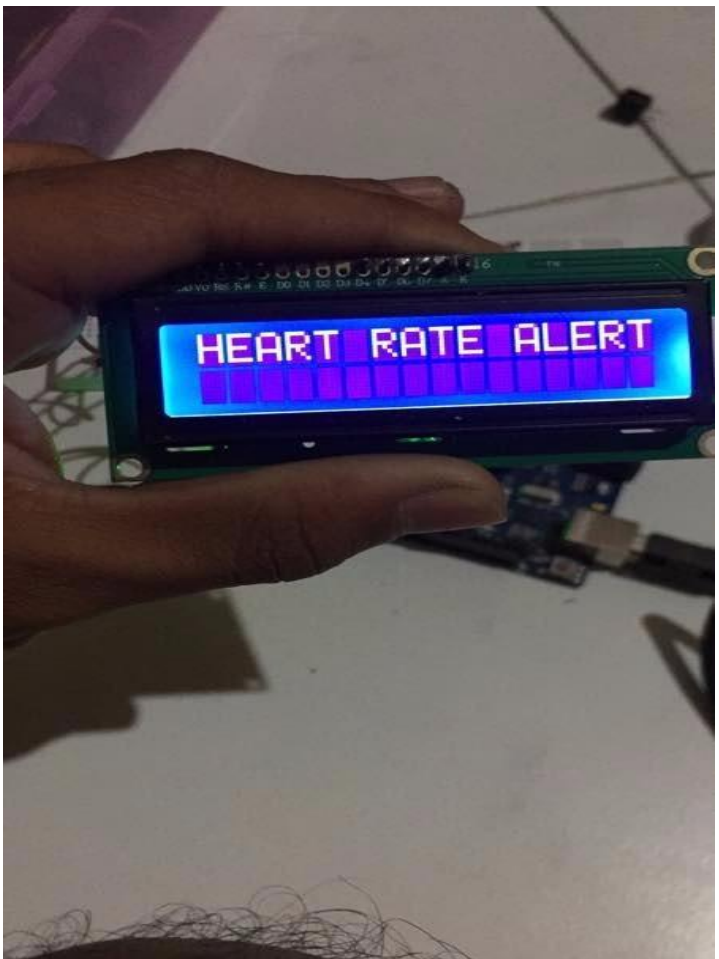

Fig. 6. Alert message display on LCD

Configured maximum range for the Human-Heartbeat which is measured, if the measured Human-Heartbeat is not within the range the LCD will display a message showing the HumanHeartbeat Alert and a buzzer to beep only for this special purpose. The buzzer will go beep if the measured HumanHeartbeat is not within the range.

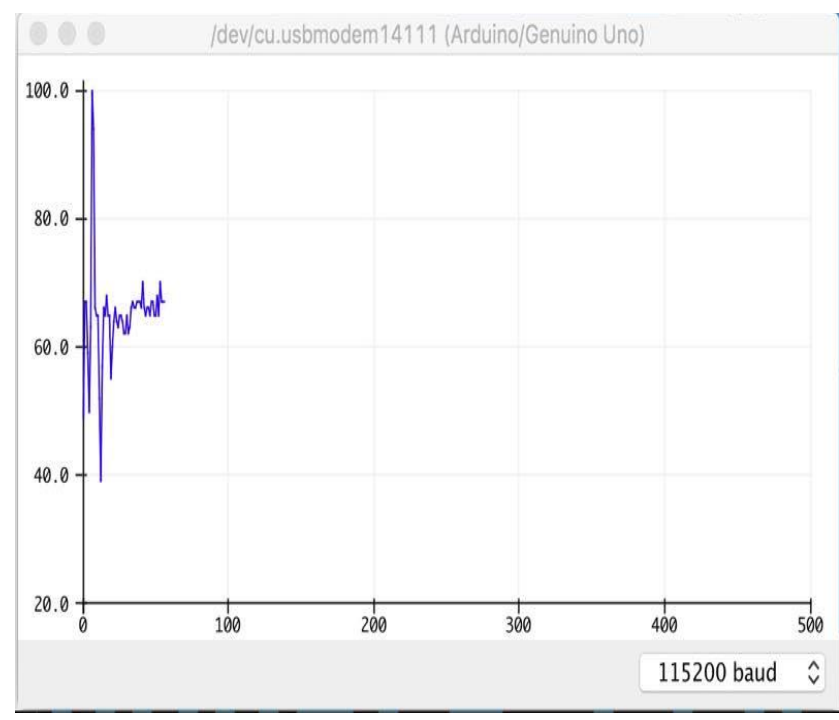

Fig. 7. Human-Heart rate graph plotted with respect to time.

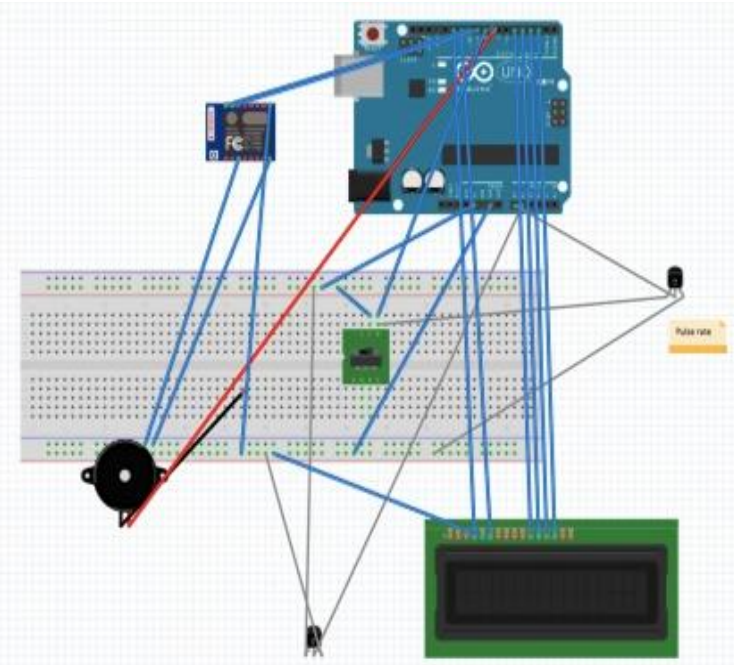

Fig. 8. pin connections of all the sensors in sensing module

\section{Field 1 Chart}

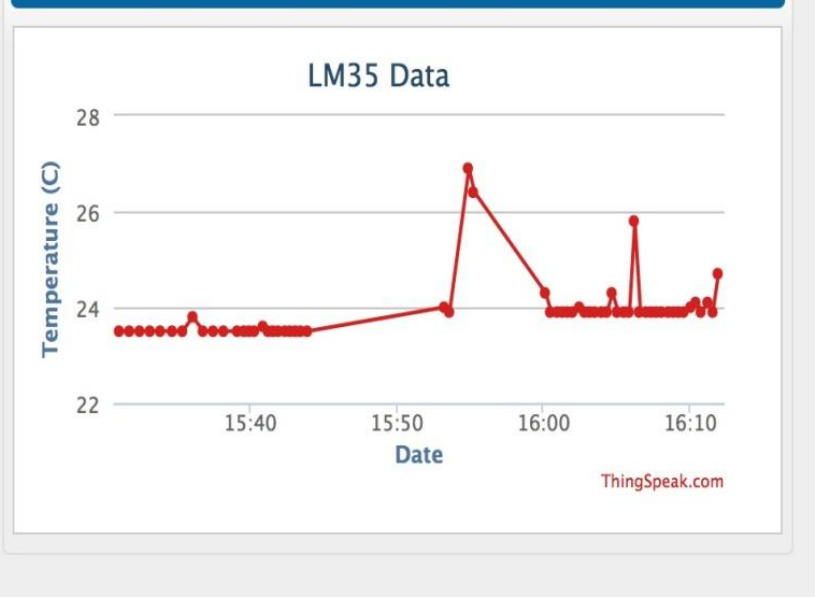

Fig. 9. Temperature graph of uploaded data on thingspeak with respect to time 


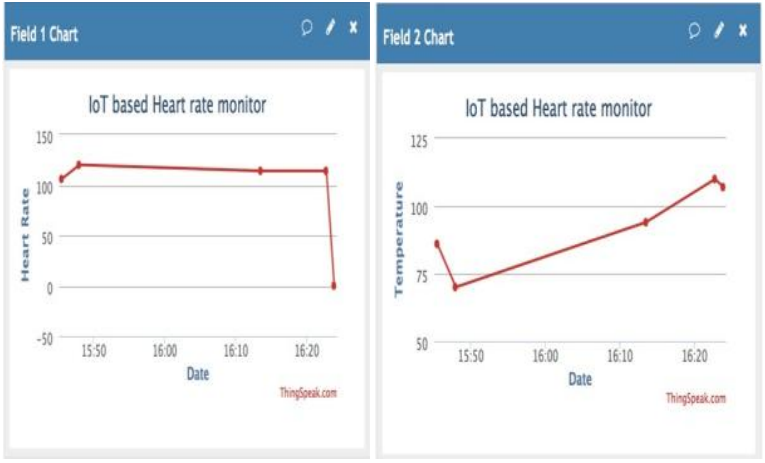

Fig. 10. Data display in form of graph

All the reading of the different test case is uploaded to the server where the data is stored, so as any person to view the patient details regularly. The readings will be updated regularly giving the scope for real time Observing of the patient. This makes easy for a doctor to check on the patient even if the patient is not in reach of a doctor.

\subsection{Experimental Results on Temperature Sensor}

LM35 is an analog temperature sensor which outputs an analog signal. Microcontrollers don't accept analog signals as their input directly. Thus, need to convert analog output signal to digital before feeding to a microcontroller's input. For this reason, use an ADC. Modern day boards like Arduino and most modern day micro controllers come with inbuilt ADC. Arduino Uno has an in-built 10-bit ADC (6 channel). Make use of this in-built ADC of Arduino to convert the analog output of LM35 to digital output. Since Arduino Uno has a 6channel inbuilt ADC, there are 6 analog input pins numbered from A0 to A5. Connect analog out of LM35 to any of these analog input pins of Arduino.

After checking all the pin connections and adding the library to the Arduino IDE and uploading the source code, run the code. The LCD screen will display the temperature in Celsius.

\section{CONCLUSION}

Developed a system that measures and detect HumanHeartbeat and body temperature of the patient, sends the data to user or server end by using microcontroller with reasonable cost and great effect. Use two different sensors and these are mainly under the control of microcontroller. For HumanHeartbeat measurement use fingertip, it's in bpm (beats per minute). These calculated rates will have stored in server by transferring through Wi-Fi module via internet. liquid crystal display (LCD) has been used to display the calculated humanheart beat rate.

To measure the human body temperature, use LM35 sensor, the measured data is given to transmitter module, it interns transfer these data to server through wireless system due to this notice avoided use of wires. Finally, the stored data in server will be displayed for further analysis by physician or specialist to provide better aid. From Experimental results, proposed system is user friendly, reliable, economical.

Further research work can be carried out for the following issues:

- In Real-time heath monitoring system using ARDUINO can be integrated or implemented in hardware using various types of sensors to detect the human-health conditions of the patients in critical sites continuous Observing of health can be made and the data's will be stored in database.

- In future, a portable Human-Health monitoring system can be designed using Arduino.

\section{REFERENCES}

[1] Mohammad Pourhomayoun, Nabil Alshurafa, Foad Dabiri, Ehsan Ardestani, Ahsan Samiee, Hassan Ghasemzadeh, Majid Sarrafzadeh, "Why Do We Need a Remote Human-Health Monitoring System? A Study on Predictive Analytics for Heart Failure Patients", JOMS, June 2011a.

[2] Ananda Mohon Ghosh, Debashish Halder, SK Alamgir Hossain, "Remote Human-Health monitoring System through IoT", 2016 5th International Conference on Informatics, Electronics and Vision (ICIEV).

[3] Mohammad Wajih Alam1, Tanin Sultana2 and Mohammad Sami Alam3," A Heartbeat and Temperature Measuring System for Remote Human-Health monitoring using Wireless Body Area Network", International Journal of Bio-Science and BioTechnology Vol.8, No.1 (2016), pp.171-190.

[4] K. Sundara Velrani, Dr.G. Geetha, "Sensor Based Healthcare Information System", 2016 IEEE International Conference on Technological Innovations in ICT For Agriculture and Rural Development.

[5] Priyanka Kakria, N. K. Tripathi, and Peerapong Kitipawang," A Real-Time Human-Health Monitoring System for Remote Cardiac Patients Using Smartphone and Wearable Sensors", International Journal of Telemedicine and Applications Volume 2015.

[6] Manisha Shelar, Jaykaran Singh, Mukesh Tiwari, "Wireless Patient Human-Health Monitoring System", International Journal of Computer Applications (0975 8887) Volume 62- No.6, January 2013.

[7] Prosanta Gope and Tzonelih Hwang, "BSN-Care: A Secure IoT-Based Modern Healthcare System Using Body Sensor Network", IEEE SENSORS JOURNAL, VOL. 16, NO. 5, MARCH 1, 2016.

[8] Prof.Y.R.Risodkar. Prof. M. K. Sangole. Amruta.R.Vankhede. Ravi.S.Medhe. Jayashri.K.Shirsat, "Web Based Human-Health monitoring System", International Journal of Advanced Research in Electronics and Communication Engineering (IJARECE) Volume 4, Issue 1, January 2015.

[9] Rajalakhshmi.S S. Nikilla," Real Time Human-Health Monitoring System using Arduino", South Asian Journal of Engineering and Technology Vol.2, No.18 (2016) 52 60.

[10] Hyung-Woo Kang, Cheol-Min Kim, Seok-Joo Koh, "ISO/IEEE 11073-based Healthcare Services over IoT Platform using 6LoWPAN and BLE: Architecture and Experimentation", 2016 International Conference on Networking and Network Applications. 